\title{
Dynamic Power Allocation for Throughput Utility Maximization in Interference-Limited Networks
}

\author{
Honghao Ju, Ben Liang, Senior Member, IEEE, Jiandong Li, Senior Member, IEEE, and Xiaoniu Yang
}

\begin{abstract}
We present an algorithm to dynamically allocate transmission power to maximize the throughput-utility in an interference-limited network under an instantaneous sum power constraint with time-varying channels. We consider the equivalent problem of maximum admission with queue stability constraint through Lyapunov optimization. The resultant non-convex minimization problem is solved by an online algorithm consisting of two components: first, successive convex approximations to randomly choose a local minimum, and second, a modified pickand-compare method for low-complexity convergence to a global minimum. We prove the optimality of this approach, derive its tradeoff between throughput-utility and delay, and demonstrate its performance advantage against existing methods.
\end{abstract}

\section{INTRODUCTION}

We consider a time-slotted interference-limited network with $M$ users. Let $\mathcal{M}:=\{(m, n)\}$ be the set of communication pairs, where $m$ and $n, 1 \leq m<M, 1 \leq$ $n \leq M$, are the transmitter and receiver, respectively. The transmission rate for $(m, n)$ at time slot $t$ is modeled as $R_{m n}(\mathbf{p}(t), \mathbf{G}(t))=\log \left(1+\Gamma \operatorname{SINR}_{m n}(t)\right)[\mathrm{bits} / \mathrm{s} / \mathrm{Hz}]$, where $\mathbf{p}(t)=\left[p_{m n}(t)\right]_{(m, n) \in \mathcal{M}}$ is the vector of transmission power allocated to $(m, n), \mathbf{G}(t)=\left[g_{m n}(t)\right]_{M \times M}$ is the matrix of channel power gain from $m$ to $n, 0<\Gamma \leq 1$ is a capacity gap from the Shannon bound, and $\operatorname{SINR}_{m n}(t)$ is given by

$$
\operatorname{SINR}_{m n}(t)=\frac{v_{m n} g_{m n}(t) p_{m n}(t)}{\sum_{(k, l) \in \mathcal{M},(k, l) \neq(m, n)} g_{k n}(t) p_{k l}(t)+\sigma^{2}},
$$

where $v_{m n}$ is the processing gain for $(m, n)$ and $\sigma^{2}$ is the noise power.

An important open problem is the optimal allocation of transmission power $p_{m n}(t)$ to maximize the sum-rate at each time slot $t$ :

$$
\max _{\mathbf{p}(t)} \sum_{(m, n) \in \mathcal{M}} R_{m n}(\mathbf{p}(t), \mathbf{G}(t))
$$

given a per-node power constraint $\sum_{n:(m, n) \in \mathcal{M}} p_{m n}(t) \leq$ $P_{m}^{\max }, \forall m$. This problem is known to be NP-hard in general [1]. Approximating in the high SINR region, the problem can be efficiently solved by geometric programming [2]. However, since the SINR depends on the power allocation decision to be made, such approximation could induce large performance loss. Other known heuristics include iterative water filling [3], successive convexification [2][4], and asymptotic Lagrange

This work was supported by National Natural Science Foundation of China (61231008,61102057), the National Basic Research Program of China (973 Program) (2009CB320404), and the Program for Changjiang Scholars and Innovative Research Team in University (PCSIRT) (IRT0852).

Honghao Ju, Jiangdong $\mathrm{Li}$, and Xiaoniu Yang are with the Information Science Institute, State Key Laboratory of Integrated Service Networks, Xidian University, China. Ben Liang is with the Department of Electrical and Computer Engineering, University of Toronto, Canada. Honghao Ju was a visiting student at the University of Toronto supported by the China Scholarship Council. duality either with small tone spacing [5] or with an infinite number of sub-carriers [6]. None of these methods guarantees a global optimum. In [7], a globally optimal solution was derived with the prismatic branch and bound method, but its computation complexity is exponential. Furthermore, all of these methods consider only a static setting where the channel gains are fixed. When the channels are time-varying, the computational complexity multiplies.

Alternatively, the problem may be recast as maximizing the time-averaged throughput, which is equivalent to maximization of the instantaneous rate asymptotically in $t$. It can be solved by employing a Lyapunov-typed cross-layer optimization technique iteratively over multiple time slots [8]. However, this technique involves the minimization of a Lyapunov drift-plus-penalty function, which is reduced to an NPhard max-weight scheduling problem. Low-complexity pickand-compare $(\mathrm{PaC})$ algorithms have been studied in [9]-[13] to iteratively compute the max-weight solution. These algorithms are proven to achieve arbitrarily near optimal throughput. However, they suffer from long transmission delays due to the unavoidable tradeoff between throughput and queue lengths. In particular, since one step of the $\mathrm{PaC}$ technique requires picking a max-weight solution that has a positive probability to be near optimal, the delay is severe when the $\mathrm{PaC}$ technique is applied to our rate maximization problem, which admits an uncountable space for power allocation.

In this paper, we consider the general problem of maximizing the throughput utility in interference-limited networks. We propose a novel algorithm that can substantially reduce the queueing delay over simple $\mathrm{PaC}$ algorithms. First, the uncountable power allocation space is reduced to a finite set of local minima by a successive convex approximation technique applied in each time slot. Then, a modified form of $\mathrm{PaC}$ is employed to dynamically extract the global optimum. The proposed algorithm is proven to efficiently solve the throughput utility maximization problem over a continuous power space, incurring a tradeoff between $1-O\left(\frac{1}{V}\right)$ utility and $O(V)$ delay for arbitrary $V>0$. We further demonstrate its performance advantage over existing alternatives.

\section{System Model And Problem Formulation}

We assume that the channel process $\mathbf{G}(t)$ is ergodic and takes value from a finite set with probability $\pi\left(\mathbf{G}^{i}\right) \triangleq$ $\operatorname{Pr}\left[\mathbf{G}(t)=\mathbf{G}^{i}\right]$. Furthermore, the power constraint induces a maximum rate $R_{\max }$, such that $R_{m n}(\mathbf{p}(t), \mathbf{G}(t)) \leq R_{\max }, \forall t$ and $(m, n) \in \mathcal{M}$. For simplicity of illustration, we initially focus on throughput maximization and later discuss the extension to throughput utility maximization in Section VI.

Instead of directly maximizing the sum of instantaneous 
rates as in (1), we maximize the sum of time-averaged rate

$$
\bar{R}_{m n}=\lim _{u \rightarrow \infty} \frac{1}{u} \sum_{t=1}^{u} R_{m n}(\mathbf{p}(t), \mathbf{G}(t)) .
$$

With only a constraint on the instantaneous power, this corresponds to an asymptotic solution to (1), i.e., for any power allocation strategy that maximizes $\sum_{(m, n) \in \mathcal{M}} \bar{R}_{m n}$, there exists a time $t_{0}$ such that $\sum_{(m, n) \in \mathcal{M}} R_{m n}(\mathbf{p}(t), \mathbf{G}(t))$ is maximized for all $t>t_{0}$.

We assume that for each communication pair $(m, n)$, $m$ always has data to send, and $Q_{m n}(t)$ is the transmission queue length at time $t$. In order to maintain queue stability, we consider an admission control strategy, where the admitted amount of data for $(m, n)$ at time $t$ is $a_{m n}(t)$. Then, the queue evolution function is expressed as $Q_{m n}(t+1)=\max \left\{Q_{m n}(t)-R_{m n}(\mathbf{p}(t), \mathbf{G}(t)), 0\right\}+a_{m n}(t)$. Furthermore, define the throughput of $(m, n)$ as $\bar{a}_{m n}=$ $\lim _{t \rightarrow \infty} \frac{1}{u} \sum_{t=1}^{u} a_{m n}(t)$.

Then our problem can be equivalently stated as

$$
\begin{array}{ll} 
& \max _{\mathbf{a}(t), \mathbf{p}(t)} \sum_{(m, n) \in \mathcal{M}} \bar{a}_{m n} \\
\text { s. t. } & \bar{a}_{m n}<\bar{R}_{m n}, \quad \forall(m, n) \in \mathcal{M}, \\
& a_{m n}(t) \leq R_{\max }, \quad \forall(m, n) \in \mathcal{M}, \\
& \sum_{n} p_{m n}(t) \leq P_{m}^{\max }, \quad \forall m, \\
& p_{m n}(t) \geq 0, \quad \forall(m, n) \in \mathcal{M},
\end{array}
$$

where $\mathbf{a}(t)=\left[a_{m n}(t)\right]_{(m, n) \in \mathcal{M}}$. In the above, (4) is required for queue stability, and (5) is an upper bound on the admission rate that does not affect the throughput optimality but is used later to improve the cleanliness of analysis ${ }^{1}$. Thus, our objective is to choose a policy for joint admission and power allocation over time, to maximize the network throughput while maintaining queue stability.

\section{EFFicient Admission AND POWER AllocAtion}

To solve the above optimization problem, a standard approach is to construct the following Lyapunov drift-pluspenalty function [8]:

$$
\begin{aligned}
& \mathbb{E}\left\{\frac{1}{2} \sum_{(m, n) \in \mathcal{M}}\left[\max \left\{Q_{m n}(t)-R_{m n}(\mathbf{p}(t), \mathbf{G}(t)), 0\right\}+a_{m n}(t)\right]^{2}\right. \\
& \left.-\frac{1}{2} \sum_{(m, n) \in \mathcal{M}} Q_{m n}^{2}(t) \mid \mathbf{Q}(t)\right\}-V \mathbb{E}\left\{\sum_{(m, n) \in \mathcal{M}} a_{m n}(t) \mid \mathbf{Q}(t)\right\},
\end{aligned}
$$

where $\mathbf{Q}(t)=\left[Q_{m n}(t)\right]_{(m, n) \in \mathcal{M}}$ and $V$ is an arbitrary positive constant. The function has the following upper bound:

$$
\begin{gathered}
|\mathcal{M}| R_{\max }^{2}+\mathbb{E}\left\{\sum_{(m, n) \in \mathcal{M}}\left(Q_{m n}(t)-V\right) a_{m n}(t)-\right. \\
\left.Q_{m n}(t) R_{m n}(\mathbf{p}(t), \mathbf{G}(t)) \mid \mathbf{Q}(t)\right\}
\end{gathered}
$$

It has been shown in [8] that any strategy that minimizes such an upper bound, in our case (8) subject to (5), (6), and (7),

\footnotetext{
${ }^{1}$ All transmission queues remain constantly backlogged under this condition, as can be seen in the admission control algorithm described in (9), so the sum input into the queues equals the sum output of the queues for any upper bound of $a_{m n}(t)$ greater than $R_{\max }$.
}

solves the original optimization problem, with $V$ as a tuning parameter that determines the tradeoff between optimization performance and queueing delay. We adopt this general approach for joint admission control and power allocation.

\section{A. Admission Control}

From the form of (8), the optimal data admission for $(m, n)$ at time $t$ is independent of power allocation:

$$
a_{m n}(t)=\left\{\begin{array}{ll}
R_{\max }, & \text { if } Q_{m n}(t)-V<0 \\
0, & \text { if } Q_{m n}(t)-V \geq 0
\end{array} .\right.
$$

\section{B. Power Allocation by Pick-and-Compare (PaC)}

To determine the optimal power to minimize the last term in (8), we have the following problem at each time $t$,

$$
\min _{\mathbf{p}(t)}\left\{\sum_{(m, n) \in \mathcal{M}}-Q_{m n}(t) \log \left(1+\Gamma \operatorname{SINR}_{m n}(t)\right)\right\},
$$

subject to power constraints (6) and (7). Note that this is a generalized form of (1), so it is at least as difficult as the original problem due to the non-convexity of $\operatorname{SINR}_{m n}(t)$.

However, instead of solving this intractable problem directly at each time slot, a random pick-and-compare algorithm [9] can be used to dynamically compute its solution over multiple time slots. Using power allocation as an example, the $\mathrm{PaC}$ algorithm consists of two steps to compute a power allocation $\mathbf{p}^{P a C}(t)$ for each $t$ :

1) A power allocation $\mathbf{p}(t)$ is randomly picked.

2) $\mathbf{p}(t)$ is compared with $\mathbf{p}^{P a C}\left(\tau_{t}\right)$, where $\tau_{t} \triangleq$ $\max \{\tau<t: \mathbf{G}(\tau)=\mathbf{G}(t)\}$, and the one that gives a smaller value to (10) for the current queue lengths $\mathbf{Q}(t)$ is chosen as $\mathbf{p}^{P a C}(t)$.

The $\mathrm{PaC}$ algorithm was first proposed in [9] for generic transmission control. With the condition that Step 1) must select an optimal solution with a positive probability, $\mathrm{PaC}$ was shown to maintain queue stability for any input vector within the network capacity region under fixed channel gains, i.e., $\tau_{t}=t-1$. It was later modified to accommodate timevarying channels in [10] and [11], and extended to throughput maximization in [12].

In power allocation problems, $\mathrm{PaC}$ is not directly applicable to a continuous power space. To guarantee a positive probability of optimality in Step 1), one may quantize power to finite levels, but such an approach is suboptimal, and it is not scalable since the decision space is exponential in the number of channels. Another technique was presented in [13] for distribution control under an SINR model, where in Step 1) a solution to (10) is picked over the continuous power space, while ensuring that it is within a constant neighborhood of the optimum with positive probability. However, near-optimal solutions generally occur with low frequency in a continuous space, which implies long queue lengths and hence large delay.

\section{SC-PaC: Creating a Finite Space for Pick-and-Compare}

We propose a new method to improve the performance of $\mathrm{PaC}$ in power allocation. It significantly reduces the decision space in a modified $\mathrm{PaC}$ algorithm, by picking from only a 
small finite set of power allocations that contains the optimal solution to (10).

Toward this end, we utilize the successive convexification (SC) approach by approximating the original problem with a series of convex optimization problems [14]. Existing SC methods applied to the SINR rate model include single condensation [2] and logarithmic approximation [4]. Here we adopt a more computationally efficient method, which also facilitates the optimality proof in Section IV.

We first rewrite (10) as

$$
\min _{\mathbf{p}(t)}\left\{\sum_{(m, n) \in \mathcal{M}} Q_{m n}(t) \log \left(\frac{\phi_{m n}(\mathbf{p}(t), \mathbf{G}(t))}{\varphi_{m n}(\mathbf{p}(t), \mathbf{G}(t))}\right)\right\},
$$

where $\phi_{m n}(\mathbf{p}(t), \mathbf{G}(t))=\sum_{k} g_{k n}(t) \sum_{(k, l) \neq(m, n)} p_{k l}(t)+$ $\sigma^{2}, \varphi_{m n}(\mathbf{p}(t), \mathbf{G}(t))=\sum_{k} g_{k n}(t) \sum_{(k, l) \neq(m, n)} p_{k l}(t)+$ $\Gamma v_{m n} g_{m n}(t) p_{m n}(t)+\sigma^{2}$. Note that the log term in (11) can be viewed as the difference of two concave functions. Then, with some initial power allocation $\mathbf{p}^{(0)}(t)$, we apply the following iterative algorithm:

a) Approximate $\log \left(\phi_{m n}(\mathbf{p}(t), \mathbf{G}(t))\right)$ with its tangent line at $\mathbf{p}^{(i)}(t)$.

b) Solve the resultant convex program and assign its minimizer to $\mathbf{p}^{(i+1)}(t)$.

c) Let $i=i+1$ and repeat from a) until convergence.

It can be shown that the sequence $\mathbf{p}^{(i)}(t)$ converges to a local minimizer of the original problem [15]. However, the SC approach cannot guarantee a global minimum. Furthermore, it is generally infeasible to obtain the set of all local minimizers in the multi-dimensional power space. Therefore, the optimality of SC, when used alone, is largely dependent on the initial point.

Fortunately, throughput optimality can be achieved by $\mathrm{PaC}$ under a milder condition that Step 1) has a positive probability to pick a global minimizer of (10). Theorem 1 below states that SC satisfies this requirement, i.e., $\operatorname{Pr}\left\{\mathbf{p}^{S C}(t)=\mathbf{p}^{*}(t)\right\}>0$, where $\mathbf{p}^{S C}(t)$ is a local minimizer derived from SC and $\mathbf{p}^{*}(t)$ is a global minimizer.

Hence, we propose an algorithm that combines SC and $\mathrm{PaC}$ :

1.1) Pick $\mathbf{p}^{(0)}(t)$ uniformly randomly from the constraint set (6) and (7).

1.2) Run SC as described above. Let $\mathbf{p}(t)=\mathbf{p}^{S C}(t)$.

2) Same as $\mathrm{PaC}$.

We label this algorithm SC-PaC. An analytical study on SC$\mathrm{PaC}$ is presented in the next section.

\section{ThroughPUT AND DELAY ANALYSIS}

In this section, we show that by reducing the decision space from uncountable to finite, $\mathrm{SC}-\mathrm{PaC}$ can substantially reduce the queueing delay toward throughput maximization. For notation simplicity, the time index $t$ is omitted when it is clear from the context. We first present Lemma 1, which is required for the proof of Theorem 1 .

Lemma 1: The set of local minima of (10) is finite.

Proof: Let $\lambda_{m}$ and $\mu_{m n}$ be Lagrange multipliers corresponding to (6) and (7), respectively. Part of the KKT conditions of (10) includes the following:

$$
\sum_{(i, j) \neq(m, n)} \frac{Q_{i j} g_{i j} g_{m n} \Gamma v_{i j} p_{i j}}{X_{i j}\left(X_{i j}+g_{i j} \Gamma v_{i j} p_{i j}\right) \ln 2}+\lambda_{m}-\mu_{m n}-
$$

$$
\begin{gathered}
\frac{Q_{m n} g_{m n} \Gamma v_{m n}}{\left(X_{m n}+g_{m n} \Gamma v_{m n} p_{m n}\right) \ln 2}=0, \quad \forall(i, j) \in \mathcal{M}, \\
\lambda_{m}\left(\sum_{n} p_{m n}-P_{m}^{\max }\right)=0, \quad \forall m,(m, n) \in \mathcal{M}, \\
\mu_{m n} p_{m n}=0, \quad \forall(m, n) \in \mathcal{M},
\end{gathered}
$$

where $X_{m n}=\sum_{(k, l) \in \mathcal{M},(k, l) \neq(m, n)} g_{k n} p_{k l}+\sigma^{2}$.

It is easy to transform (12) into a polynomial function by multiplying out the denominators, and (13) and (14) are both polynomials. Then, since there are an equal number of variables and equations, and the number of distinct monomials is finite, by Khovanskii's Theorem [16], the number of real roots of (12), (13), and (14) is finite. This implies that the set of local minima is finite.

Intuitively, since SC randomly chooses from a finite set of local minimizers by Lemma 1 , as long as the choice is unbiased in some sense, a global minimizer will be chosen with positive probability. The following theorem formalizes this observation.

Theorem 1: There exists some $\rho>0$ such that $\operatorname{Pr}\left\{\mathbf{p}^{S C}(t)=\mathbf{p}^{*}(t)\right\} \geq \rho$.

Proof: Let $Z(\mathbf{p})=\sum_{(m, n) \in \mathcal{M}} Q_{m n} \log \left(\frac{\phi_{m n}(\mathbf{p}, \mathbf{G})}{\varphi_{m n}(\mathbf{p}, \mathbf{G})}\right)$, and let $\hat{Z}^{(i)}(\mathbf{p})$ be its convex approximation in the $i$ iteration of the SC algorithm, induced by the point $\mathbf{p}^{(i)}$. By the strict concavity of $\log \left(\phi_{m n}(\mathbf{p}(t), \mathbf{G}(t))\right)$ and the definition of a tangent line, we have $Z^{(i)}(\mathbf{p}) \geq Z(\mathbf{p})$, with equality if and only if $\mathbf{p}=\mathbf{p}^{(i)}$.

Let $\mathcal{C}_{k}$ be a neighborhood where the $k$ th local minimizer $\mathbf{p}_{k}$ is the only minimizer. Let $\mathcal{H}_{k}$ be the set of local maximizers in $\mathcal{C}_{k}$, and we define a minimum-maximizer $\tilde{\mathbf{p}}_{k}=$ $\operatorname{argmin}_{\mathbf{p} \in \mathcal{H}_{k}}\{Z(\mathbf{p})\}$. Consider the region $\mathcal{U}_{k}=\left\{\mathbf{p} \in \mathcal{C}_{k}\right.$ : $\left.Z(\mathbf{p})<Z\left(\tilde{\mathbf{p}}_{k}\right)\right\}$. Suppose $\mathbf{p}^{(i)} \in \mathcal{U}_{k}$ but $\mathbf{p}^{(i+1)} \notin \mathcal{U}_{k}$. Then there always exists some $\mathbf{p}^{\prime}=\alpha \mathbf{p}^{(i)}+(1-\alpha) \mathbf{p}^{(i+1)}$, $0 \leq \alpha<1$, such that $Z\left(\mathbf{p}^{\prime}\right)=Z\left(\tilde{\mathbf{p}}_{k}\right)$. Since $\mathbf{p}^{(i+1)}$ is a global minimizer of $\hat{Z}^{(i)}(\mathbf{p})$, we have $\hat{Z}^{(i)}\left(\mathbf{p}^{(i+1)}\right) \leq$ $\hat{Z}^{(i)}\left(\mathbf{p}^{(i)}\right)=Z\left(\mathbf{p}^{(i)}\right)<Z\left(\tilde{\mathbf{p}}_{k}\right)$. Then, by convexity, we have $\hat{Z}^{(i)}\left(\mathbf{p}^{\prime}\right)<\alpha \hat{Z}^{(i)}\left(\mathbf{p}^{(i)}\right)+(1-\alpha) \hat{Z}^{(i)}\left(\mathbf{p}^{(i+1)}\right)<Z\left(\tilde{\mathbf{p}}_{k}\right)$. It follows that $\hat{Z}^{(i)}\left(\mathbf{p}^{\prime}\right)<Z\left(\mathbf{p}^{\prime}\right)$, which creates a contradiction. Hence, with any starting point $\mathbf{p}^{(0)} \in \mathcal{U}_{k}$, the sequence of SC minimizers stays inside $\mathcal{U}_{k}$, so they must converge to $\mathbf{p}_{k}$.

Since the number of local minima of (10) is finite by Lemma 1 , there exists a constant $\zeta>0$ such that $\left|\mathcal{U}_{k}\right| \geq \zeta, \forall k$. In particular, consider the $\mathcal{U}_{k}$ that contains $\mathbf{p}^{*}$. Let $\mathcal{P}$ be the feasible set defined by (6) and (7). It then follows that, when the initial point of $\mathrm{PC}$ is uniformly randomly selected in $\mathcal{P}$, $\operatorname{Pr}\left\{\mathbf{p}^{S C}(t)=\mathbf{p}^{*}(t)\right\} \geq \frac{\left|\mathcal{U}_{k}\right|}{|\mathcal{P}|}>0$.

Theorem 1 can be used to lower bound the throughput of $\mathrm{SC}-\mathrm{PaC}$, as stated in Theorem 2.

Theorem 2: SC-PaC provides the following throughput performance guarantee:

$$
\sum_{(m, n) \in \mathcal{M}} \bar{a}_{m n} \geq \bar{A}^{*}-\frac{|\mathcal{M}| R_{\max }^{2}}{V}-\frac{2|\mathcal{M}| R_{\max }^{2}}{\rho V \min _{i}\left\{\pi\left(\mathbf{G}^{i}\right)\right\}},
$$

where $\bar{A}^{*}$ is the global maximum of (3) - (7).

Proof: Due to space limitation and to avoid redundancy with existing literature, we omit some details in the standard algebraic manipulation of (8). Interested readers are referred to similar proof techniques used in [10], [11], [12]. 


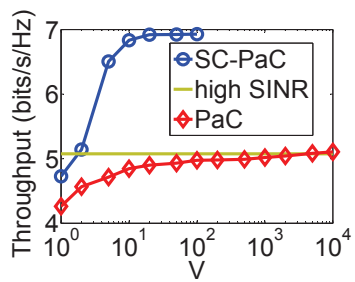

(a) Sum throughput vs. V

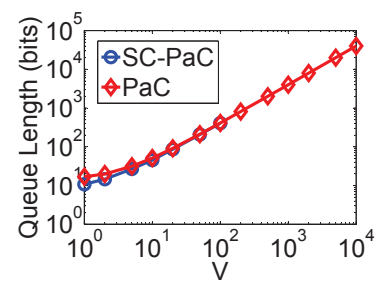

(b) Sum queue length vs. V
Fig. 1. Network performance comparison.

In particular, similar to the proof of Theorem 9 in [12], it can be shown that $\mathbb{E}\left[\sum_{(m, n)} Q_{m n}(t)\left(R_{m n}\left(\mathbf{p}^{*}(t), \mathbf{G}(t)\right)-\right.\right.$ $\left.\left.R_{m n}\left(\mathbf{p}^{P a C}(t), \mathbf{G}(t)\right)\right) \mid \mathbf{Q}(t)\right] \leq \frac{2|\mathcal{M}| R_{\max }^{2}}{\rho \min _{i}\left\{\pi\left(\mathbf{G}^{i}\right)\right\}}$. Then (15) follows from Theorem 4.8 in [8].

Meanwhile, the admission control strategy in (9) dictates that each queue does not exceed $V+R_{\max }$, so an upper bound to the sum queue length is $\sum_{(m, n) \in \mathcal{M}} Q_{m n}(t) \leq|\mathcal{M}|(V+$ $\left.R_{\max }\right)$. Hence, SC-PaC achieves a tradeoff between $1-O\left(\frac{1}{V}\right)$ throughput and $O(V)$ delay. This means that with a large $V$, we can drive the throughput arbitrarily closed to its optimum value, while incurring a linearly increasing delay.

Note that the term $\frac{2|\mathcal{M}| R_{\max }^{2}}{\rho V \min _{i}\left\{\pi\left(\mathbf{G}^{i}\right)\right\}}$ in (15) can be viewed as a cost incurred in exchange for reducing the complexity of power allocation. It is inversely proportional to $\rho V$, which is a general condition for $\mathrm{PaC}$ methods. However, as SC$\mathrm{PaC}$ reduces the decision space for power allocation from an uncountable continuum to a finite set, it significantly increases $\rho$ over conventional $\mathrm{PaC}$, leading to drastic increase in throughput for any given $V$ or drastic decrease in $V$ for any given throughput requirement.

\section{Numerical Evaluation}

We evaluate the performance of SC-PaC through simulation. Comparison is made with the conventional $\mathrm{PaC}$ approach used in [10]-[13] as described in Section III, and with geometric programming assuming high SINR as in [2].

We use as an example the downlink of a cellular system with one transmitter and four receivers. The channel amplitudes are modeled as i.i.d. Rayleigh random variables with unit average power, which are quantized into six equal-probability states $\{0.280,0.535,0.734,0.937,1.183,1.649\}$. We normalize the noise power to 1 and set $P_{m}^{\max }=20, v_{0 m}=5$, and $\Gamma=1$, which gives $R_{\max }=8.1 \mathrm{bps} / \mathrm{Hz}$.

From Figs. 1(a), we see that the throughput of $\mathrm{SC}-\mathrm{PaC}$ is substantially higher than that of $\mathrm{PaC}$ for the same $V$. It also out performs geometric programming when $V \geq 2$, while $\mathrm{PaC}$ requires $V>5000$, incurring extremely high delay. Furthermore, there is no significant improvement in the SC$\mathrm{PaC}$ throughput when $V>20$, suggesting that the global optimum can be approached without too much delay. From Fig. 1(b), we see that $\mathrm{SC}-\mathrm{PaC}$ and $\mathrm{PaC}$ induce similar queue backlog for $V>20$, roughly at $|\mathcal{M}| V$ as expected from Section IV. Hence, the throughput improvement by SC-PaC is achieved without delay penalty.

\section{EXTENSION TO THROUGHPUT UTILITY MAXIMIZATION}

The proposed method can be easily extended to maximize the sum of throughput utility, by replacing $\bar{a}_{m n}$ with $U\left(\bar{a}_{m n}\right)$ in (3), where $U(\cdot)$ is some concave non-decreasing function. This is useful, for example, when fairness among the communication pairs is considered. The general form of such extension is given in [8], which shows that the problem can be transformed into maximizing the sum of time-averaged utility of some instantaneous rate $b_{m n}(t)$, such that $\lim _{t \rightarrow \infty} \frac{1}{u} \sum_{t=1}^{u} b_{m n}(t) \leq \bar{a}_{m n}$. This allows a similar Lyapunov drift-plus-penalty formulation, with an additional virtual queue $H_{m n}(t)$ for each $(m, n)$, with updating function $H_{m n}(t+1)=\max \left\{H_{m n}(t)-a_{m n}(t), 0\right\}+b_{m n}(t)$.

Then, it can be shown that optimal admission is modified from (9) to $a_{m n}(t)=R_{\max }$, if $Q_{m n}(t)-H_{m n}(t)<0$, and 0 otherwise, and an optimal $b_{m n}(t)$ can be obtained from minimizing $H_{m n}(t) b_{m n}(t)-V U\left(b_{m n}(t)\right)$, which is a convex function of $b_{m n}(t)$. Furthermore, the power allocation remains as in (10), so that SC-PaC can be applied without further modification. Finally, it can be proven similarly that we have a tradeoff between $1-O\left(\frac{1}{V}\right)$ utility and $O(V)$ delay.

\section{REFERENCES}

[1] Z.-Q. Luo and S. Zhang, "Dynamic spectrum management: Complexity and duality," IEEE J. Sel. Topics Signal Process., vol. 2, no. 1, pp. 5773, Feb. 2008

[2] M. Chiang, C. Tan, D. Palomar, D. O'Neill, and D. Julian, "Power control by geometric programming," IEEE Trans. Wireless Commun., vol. 6, no. 7, pp. 2640-2651, 2007.

[3] W. Yu, G. Ginis, and J. Cioffi, "Distributed multiuser power control for digital subscriber lines," IEEE J. Sel. Areas Commun., vol. 20, no. 5, pp. 1105-1115, 2002.

[4] J. Papandriopoulos and J. Evans, "Scale: A low-complexity distributed protocol for spectrum balancing in multiuser DSL networks," IEEE Trans. Inf. Theory, vol. 55, no. 8, pp. $3711-3724,2009$.

[5] R. Cendrillon, W. Yu, M. Moonen, J. Verlinden, and T. Bostoen, "Optimal multiuser spectrum balancing for digital subscriber lines," IEEE Trans. Commun., vol. 54, no. 5, pp. 922-933, 2006.

[6] W. Yu and R. Lui, "Dual methods for nonconvex spectrum optimization of multicarrier systems," IEEE Trans. Commun., vol. 54, no. 7, pp. 13101322, 2006.

[7] Y. Xu, T. Le-Ngoc, and S. Panigrahi, "Global concave minimization for optimal spectrum balancing in multi-user DSL networks," IEEE Trans. Signal Process., vol. 56, no. 7, pp. 2875-2885, 2008.

[8] M. Neely, Stochastic network optimization with application to communication and queueing systems. Morgan \& Claypool Publishers, 2010.

[9] L. Tassiulas, "Linear complexity algorithms for maximum throughput in radio networks and input queued switches," in Proc. IEEE INFOCOM, vol. 2, 1998, pp. 533-539.

[10] A. Eryilmaz, R. Srikant, and J. Perkins, "Stable scheduling policies for fading wireless channels," IEEE/ACM Trans. Netw., vol. 13, no. 2, pp. 411-424, 2005.

[11] M. Lotfinezhad, B. Liang, and E. S. Sousa, "On stability region and delay performance of linear-memory randomized scheduling for timevarying networks," IEEE/ACM Trans. Netw., vol. 17, no. 6, pp. 18601873, Dec. 2009

[12] P. Chaporkar and S. Sarkar, "Stable scheduling policies for maximizing throughput in generalized constrained queueing systems," IEEE Trans. Autom. Control, vol. 53, no. 8, pp. 1913-1931, 2008.

[13] H.-W. Lee, E. Modiano, and L. B. Le, "Distributed throughput maximization in wireless networks via random power allocation," IEEE Trans. Mobile Comput., vol. 11, no. 4, pp. 577 -590, 2012.

[14] B. Marks and G. Wright, "A general inner approximation algorithm for nonconvex mathematical programs," Operations Research, pp. 681-683, 1978.

[15] S. Boyd, "Convex optimization II," Lecture Notes, 2008. [Online]. Available: http://www.stanford.edu/class/ee364b/lectures.html

[16] B. Sturmfels, "Polynomial equations and convex polytopes," The American mathematical monthly, vol. 105, no. 10, pp. 907-922, 1998. 